\title{
Postoperative complications and quality of life in patients with pituitary adenoma
}

\author{
Jiajia Zhang ${ }^{1 \#}$, Ya Wang ${ }^{1 \#}$, Xiuqun $\mathrm{Xu}^{2}$, Yudan Gu ${ }^{1}$, Fei Huang ${ }^{1}$, Min Zhang ${ }^{1}$ \\ ${ }^{1}$ Department of Neurosurgery, Affiliated Hospital of Nantong University, Nantong, China; ${ }^{2}$ Department of Nursing, Affiliated Hospital of Nantong \\ University, Nantong, China \\ Contributions: (I) Conception and design: J Zhang; (II) Administrative support: X Xu; (III) Provision of study materials or patients: Y Gu; (IV) \\ Collection and assembly of data: Y Wang, X Xu; (V) Data analysis and interpretation: Y Gu; (VI) Manuscript writing: All authors; (VII) Final \\ approval of manuscript: All authors. \\ "These authors contributed equally to this work. \\ Correspondence to: Xiuqun Xu. Department of Nursing, Affiliated Hospital of Nantong University, Nantong 226001, China. \\ Email: 983809906@qq.com; Yudan Gu. Department of Neurosurgery, Affiliated Hospital of Nantong University, Nantong 226001, China. \\ Email: guyd006@163.com.
}

Background: Pituitary adenomas (PAs) are tumors that arise from the cells of the anterior pituitary gland. PAs are the most common tumors to occur in the sella area and the third most common intracranial neoplasm in surgery. Due to developments such as microscopy and neuroendoscopy, the curative effect of PA surgery continues to improve. However, postoperative complications may still occur. PA surgery can result in iatrogenic trauma, which is a primary cause of cerebrospinal fluid (CSF) rhinorrhea. PA surgery can affect the posterior pituitary gland, causing decreased antidiuretic hormone secretion and increased urine output, which can lead to hyponatremia. The nasal cavity and sphenoid sinuses may also be damaged during PA surgery, causing the sphenoethmoidal recess to be blocked. This increases the risk of infection. The purpose of this study was to analyze quality of life in PA patients post-resection, and to provide references for the development of early targeted risk assessment programs and intervention measures.

Methods: We selected patients who underwent neuroendoscopic transnasal PA resection at the Affiliated Hospital of Nantong University between January 2017 and October 2019. These patients' hospital records were retrospectively obtained, including details of any postoperative complications. The patients were followed up by telephone 12 weeks following discharge, and we used the EuroQol 5 Dimensions (EQ-5D) health scale to assess the patients' quality of life.

Results: At follow-up 3 months after discharge, 68 people (41.72\%) had experienced at least one common complication, including 7 cases of infection (4.29\%), 56 cases of hypopituitarism (34.36\%), 18 cases of CSF rhinorrhea (11.04\%), 28 cases of diabetes insipidus (17.18\%), and 25 cases of hyponatremia (15.34\%). Patients that experienced complications reported having more serious problems on the dimensions of the EQ-5D descriptive system (mobility, self-care, usual activities, pain/discomfort, and anxiety/depression) than patients without complications. The scores of patients with complications on the EQ-5D visual analog scale (EQ-VAS) were lower than that of patients without complications.

Conclusions: Postoperative complications are negatively associated with quality of life in PA patients. Actively preventing common complications could therefore improve these patients' quality of life and reduce their burden of disease.

Keywords: Pituitary adenoma (PA); complication; EuroQol 5 Dimensions (EQ-5D); EQ-5D visual analog scale (EQ-VAS); quality of life; nursing care

Submitted Aug 06, 2020. Accepted for publication Sep 27, 2020.

doi: $10.21037 /$ gs-20-690

View this article at: http://dx.doi.org/10.21037/gs-20-690 


\section{Introduction}

Pituitary adenomas (PAs) are tumors that arise from the cells of the anterior pituitary gland. These tumors are the most common to occur in the sella area and the third most common tumor treated with brain surgery. PAs account for approximately $10 \%$ to $25 \%$ of intracranial tumors identified in a clinical setting (1). These tumors are hormonesecreting tumors: $32 \%$ to $66 \%$ of PAs are prolactinomas that secrete excessive levels of prolactin (PRL), $8 \%$ to $16 \%$ secrete growth hormone $(\mathrm{GH}), 2 \%$ to $6 \%$ secrete adrenocorticotropic hormone (ACTH), and 1\% secretes thyroid stimulating hormone (TSH). PAs can also be nonfunctioning tumors, which account for 15-54\% (2).

Though most PAs are benign, approximately $0.1 \%$ to $0.2 \%$ are aggressive tumors. The prognosis of PAs is directly related to their degree of malignancy (3). The clinical manifestations of PAs are primarily symptoms of endocrine disorders and the compression of surrounding tissue structures by the tumor. The goal of treatment for PA is to eliminate tumor compression, protect nerve function, promote a return to normal hormonal and metabolic functioning, and to improve patient quality of life (4).

Surgical intervention is the principal and most effective therapy for PAs. Due to technological developments such as microscopy and neuroendoscopy, the curative effect of PA surgery continues to improve. Despite this, postoperative complications may still occur. PA surgery can result in iatrogenic trauma, which is one of the primary causes of cerebrospinal fluid (CSF) rhinorrhea (5). PA surgery also affects the posterior pituitary to varying degrees. This decreases antidiuretic hormone secretion and increases urine output, which can lead to hyponatremia (6). Technological advances have led to the development of microscopic and neuroendoscopic surgery for transnasal and transsphenoidal PAs. Although these surgeries are widely used, microscopic surgery involves expanding the nasal cavity and separating the nasal septum. Pushing the septum to one side can damage the nasal cavity and sphenoid sinuses during surgery, which can obstruct the sphenoethmoidal recess. This increases the risk of infection (7).

Complications following PA resection can be serious. For example, Li et al. (8) found that hypopituitarism occurs in $3.6 \%$ to $13.6 \%$ of $\mathrm{PA}$ resection patients. Patients with mild cases of hypopituitarism show indifference, lethargy, and feel hazy, while those with severe cases may experience critical symptoms, such as coma. For many PA patients, the symptoms they experience prior to tumor resection, including fatigue, infertility, daytime sleepiness, sleep disturbance, low vision, changes in appearance, depression, and anxiety, continue post-surgery $(9,10)$. There are numerous postoperative complications that negatively impact $\mathrm{PA}$ resection patients. Following surgery, various complications can have a serious impact on the patients. If the patients are unable to cope with these complications due to inability to adapt, it may lead to a negative mentality and unwillingness to receive follow-up. As a result, follow-up rates and treatment compliance for these patients are poor. This poor compliance delays treatment and facilitates disease progression, which can seriously impact patient quality of life.

Quality of life emerged as a subject of scientific research in the 1940s and usually refers to health-related quality of life (HRQOL), though there is no widely accepted unambiguous definition. In 1993, the World Health Organization (WHO) Quality of Life Research Group (5) defined quality of life as the experience of individuals of different cultures and value systems with regard to their goals, expectations, standards, and living conditions as related to their concerns. Schipper (11) and others, however, believe that quality of life is a daily, practical, and functional description of an individual's physical, psychological, and social responses to the diseases they have and the treatment they receive. Levi (12) and others have suggested that quality of life is a comprehensive measurement of the physical, psychological, and social adaptation status of an individual or a group. Wan (13) and others believe that quality of life is a subjective perception and experience of a patient's physical,

psychological, and social status as a result of disease and treatment. Quality of life is a subjective feeling primarily dependent on the judgment of patients. It is also a dynamic process: individual subjective feelings will change with time, stress, and implementation of coping strategies (14). Quality of life has become a widely recognized end-point indicator for assessing treatment and care in oncology. Clinical oncologists emphasize that quality of life means to create conditions for patients to enjoy a better life.

There has been limited research of the correlations between postoperative complications in PA resection patients and quality of life post-discharge. The aim of this study was to analyze the current quality of life and factors that influence it in patients that had undergone PA resection, as well as to screen for high-risk groups, s and provide references for the formulation of early and targeted risk assessment plans and intervention measures. Significant steps to improve quality of life for patients following PA 


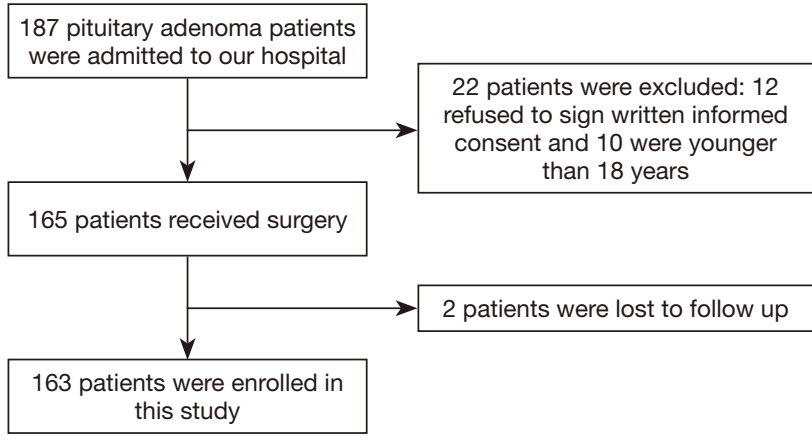

Figure 1 Study flow chart.

resection are yet to be taken. Patients must be provided with more effective psychological interventions, discharge support, and guidance for coping with cognitive disease. Additionally, the quality of discharge services must be improved and the continuity of medical care services as a whole enhanced. An established and in-depth understanding of the relationship between coping mechanisms and quality of life in populations of differing cultural backgrounds has particular instructive significance for the development of nursing practices. We present the following article in accordance with the STROBE reporting checklist (available at http://dx.doi.org/10.21037/gs-20-690).

\section{Methods}

\section{Subjects}

A total of 163 patients that underwent neuroendoscopic transnasal PA resection at the Affiliated Hospital of Nantong University between January 2017 and October 2019 were selected for this study. Case report forms were used to record the patients' complications throughout hospitalization. Telephone follow-ups were conducted 12 weeks following discharge during which the patients' quality of life was measured using the EuroQol 5 Dimensions (EQ-5D) health scale. Figure 1 depicts a flow chart of the stages of this study.

The inclusion criteria for this study were as follows: (I) patients that are adults aged more than 18 years that received neuroendoscopic transnasal PA resection; (II) patients that are conscious and can cooperate to complete the study questionnaire; (III) patients that can read, write, and are able to communicate with study personnel; (IV) patients that agree to participate and sign written informed consent (see Figure 1).
The exclusion criteria were the following: (I) patients with a history of malignant tumors other than PA; (II) psychiatric patients or patients with other diseases that may impact the observable curative effects of PA resection; (III) patients with a severe disability, or that are affected by major surgery, acute infection, acute heart failure, severe cerebrovascular sequelae, et cetera.

The study was conducted in accordance with the Declaration of Helsinki (as revised in 2013). This study was approved by the ethics committee of the Affiliated Hospital of Nantong University ([2017] 02165) and obtained signed informed consent from all patients.

\section{Data collection}

An Excel electronic data collection form (Microsoft China Co., Ltd., Beijing, China) was used to collect patient data. Two senior nurses were selected as study assistants within the hospital. These nurses both had bachelor's degrees or higher and had been working in the hospital for at least 2 years. They were given uniform onsite training for this role prior to the commencement of the study. This training covered the processes of the investigation and use of the electronic data collection platform.

Following patient enrollment in the study, these study assistants recorded general patient information, such as sex, age, and education level. They also recorded clinical information, such as tumor size and pathological type.

Twelve weeks after the patients were discharged or transferred, telephone follow-ups were carried out during which the patients' complications and quality of life were assessed. Five common postoperative complications were investigated: infection, hypopituitarism, CFS rhinorrhea, diabetes insipidus, and hyponatremia. Additionally, the patients responded to each item on the EQ-5D, their scores recorded by the study assistants.

To ensure the collected data were true, reliable, and complete, medical record quality control and onsite quality control was adopted. Medical record quality control involved checking the consistency of information recorded in the data collection form with medical records, and onsite quality control involved determining whether information recorded in the data collection form matched the patients' condition as determined by medical assessment.

\section{EQ-5D descriptive system}

The EQ-5D is one of the primary measures currently used 


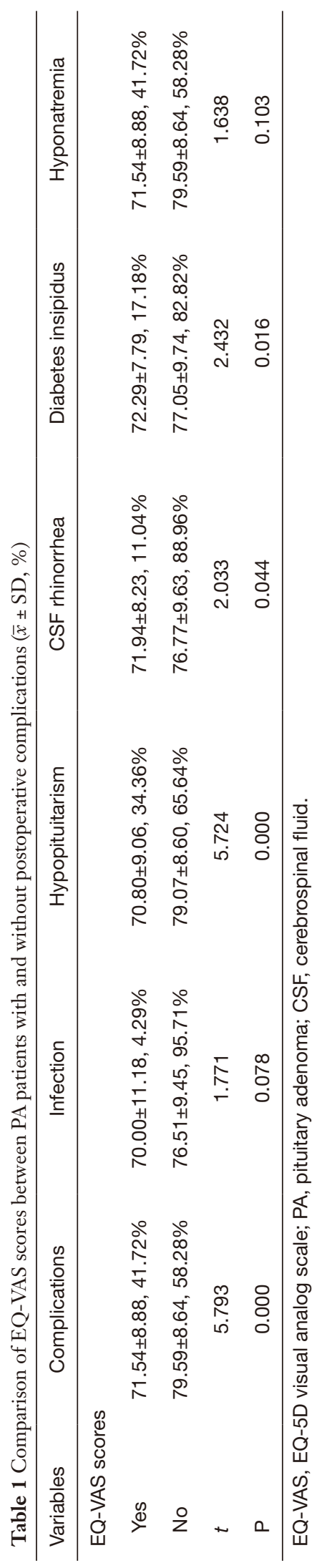

to assess HRQOL. It is frequently used in population health surveys, and this scale has been confirmed to have good validity and reliability, Kim et al. $(15,16)$ completed the reliability and validity study of the EQ-5D-3L in measuring the quality of life of 893 patients with 30 different types of cancer in South Korea in 2011, and completed the EQ5D-5L in the same year. Pickard et al. (17) completed the quality of life measurement of 424 patients with 11 types of cancer in the United States in 2007, and evaluated the reliability and validity of the two response systems in the same year. The EQ-5D descriptive system assesses five dimensions that reflect different states of health: mobility, self-care, usual activities, pain/discomfort, and anxiety/ depression. Mobility, self-care, and pain/discomfort reflect the patients' physical health, while daily activities reflect social functioning and anxiety/depression reflects the state of patients' mental health. There are three possible responses for each dimension: no problems, some problems, and extreme problems. During telephone follow-up, the study assistants recorded the patients' responses for these 5 dimensions by using the questions as part of their conversation.

\section{EuroQol visual analog scale (EQ-VAS)}

The EQ-VAS is a component of the EQ-5D and is a vertical visual analog scale, the lowest point on the scale being 0 , the poorest health imaginable, and the highest point being 100 , the best health imaginable. This is a quantitative measure that reflects the patient's personally perceived state of health (18). The EQ-VAS supplements the assessment of quality of life obtained by the EQ-5D descriptive system and is a single measure that reflects the patient's total quality of life. During the telephone followup, the investigator will introduc the scoring standards and guide the patients to evaluate their health and score. A comparison of EQ-VAS scores between patients with and without complications following PA resection is shown in Table 1.

\section{Statistical analysis}

SPSS Statistics 22.0 (SPSS Inc., Chicago, Illinois, USA) was used for the statistical analysis of all data. The continuous data are expressed as mean \pm standard deviation (SD). Independent sample $t$-tests were used to compare groups, and paired sample $t$-tests were used for intra-group comparisons. Discrete data are expressed as number of 
cases and rate (\%), and chi-squared $\left(\chi^{2}\right)$ tests were used to compare this data between groups. Statistical significance was set at $\mathrm{P}<0.05$.

\section{Results}

\section{General clinical data}

Of these 163 patients, 78 were male (47.9\%) and 85 were female $(52.1 \%)$.

Using a new method of age segmentation proposed by WHO, patients were divided into three age groups. The young age group (aged 45 or younger) contained 72 patients (44.17\%), the middle-aged group (aged 46 to 60 ) contained 39 patients $(23.93 \%)$, and the elderly group (aged 60 or older) contained 52 patients (31.90\%).

In terms of education levels, 65 patients (39.88\%) had an elementary school education or less, 80 patients (49.08\%) had a middle school/technical school education, and 18 patients $(11.04 \%)$ had a college degree or more. With regard to employment status, 90 patients $(55.21 \%)$ were employed and 73 (44.79\%) were not employed.

The patients' PAs were divided by size into microadenomas (less than $10 \mathrm{~mm}$ ), macroadenomas (equal to or more than $10 \mathrm{~mm}$ ), and giant adenomas (equal to or more than $40 \mathrm{~mm}$ ). Of these patients, 31 had microadenomas (19.02\%), 126 had macroadenomas (77.30\%), and 6 had giant adenomas (3.68\%).

PAs were also divided into groups by pathology: prolactinomas, GH-secreting PAs, ACTH-secreting PAs, gonadotropin (Gn)-secreting PAs, and non-functioning tumors. Of the 163 patients, 48 had prolactinomas (29.45\%), 18 had GH-secreting PAs (11.04\%), 12 had ACTH-secreting PAs (7.36\%), 17 had Gn-secreting PAs $(10.43 \%)$, and 68 had non-functioning tumors $(41.72 \%)$.

A total of 68 patients $(41.72 \%)$ of the 163 enrolled experienced postoperative complications during the followup period. Of these 68 patients, 7 experienced infection (4.29\%), 56 had hypopituitarism (34.36\%), 18 had CSF rhinorrhea (11.04\%), 28 had diabetes insipidus (17.18\%), and 25 had hyponatremia (15.34\%). A total of 21 patients $(12.88 \%)$ relapsed during the follow-up period.

Of these parameters, age and tumor size were found to have a significant impact on whether patients developed complications $(\mathrm{P}<0.05)$. Older patients and patients with larger tumor sizes may be a high-risk group for developing complications. The patients' clinical data are set out in Table 2.

\section{Effect of complications on EQ-5D descriptive system scores}

The scores obtained using the EQ-5D descriptive system are shown in Table 3. There were significant associations found between the presence of complications and the EQ$5 \mathrm{D}$ scores for all dimensions $(\mathrm{P}<0.05)$.

Table 4 sets out the relationships between postoperative complications (infection, hypopituitarism, CSF rhinorrhea, diabetes insipidus, and hyponatremia) and patient scores on the EQ-5D descriptive system. Hypopituitarism and CSF rhinorrhea were found to be correlated with every score on EQ-5D dimension $(\mathrm{P}<0.05)$, but the presence of hyponatremia had no significant effect on any EQ-5D scores $(\mathrm{P}>0.05)$.

\section{Comparison of EQ-VAS scores between patients with and without postoperative complications}

The mean \pm SD EQ-VAS score for patients experiencing at least one complication was $71.54 \pm 8.88$; for patients with infection it was $70.00 \pm 11.18$; for patients with hypopituitarism it was $70.80 \pm 9.06$; for patients with CSF rhinorrhea it was $71.94 \pm 8.23$; for patients with diabetes insipidus it was $72.29 \pm 7.79$; and for patients with hyponatremia it was $71.54 \pm 8.88$. The mean \pm SD EQ-VAS score for patients not experiencing any complications was $9.59 \pm 8.64$; for patients without infection it was $76.51 \pm 79.45$; for patients without hypopituitarism it was $79.07 \pm 8.60$; for patients without CSF rhinorrhea it was $76.77 \pm 9.63$; for patients without diabetes insipidus it was $77.05 \pm 9.74$; and for patients without hyponatremia it was $79.59 \pm 8.64$. The presence of at least one complication $(\mathrm{P}=0.000)$, hypopituitarism $(\mathrm{P}=0.000)$, CSF rhinorrhea $(\mathrm{P}=0.044)$, and diabetes insipidus $(\mathrm{P}=0.016)$ were found to be significantly associated with EQ-VAS scores.

\section{Discussion}

PAs are common tumors of the central nervous system that predominantly occur in people aged between 30 and 50 years (19). These tumors account for approximately $10 \%$ of intracranial tumors, and the majority of PAs are benign. This condition is most commonly treated with surgery, which can produce postoperative complications for some patients. The primary complications that occur following PA resection are CSF rhinorrhea, diabetes insipidus, infection, hypopituitarism, and hyponatremia. This study found that postoperative complications have a significant 
Table 2 General clinical data (n, \%)

\begin{tabular}{|c|c|c|c|c|}
\hline Variables & Complications $(n=68)$ & No complications ( $n=95$ ) & $\chi^{2}$ value & $P$ value \\
\hline Female & $38,55.88 \%$ & $47,49.47 \%$ & & \\
\hline Male & $30,44.12 \%$ & $48,50.53 \%$ & & \\
\hline Age & & & 6.914 & 0.032 \\
\hline $46-60$ & $21,30.88 \%$ & $18,18.95 \%$ & & \\
\hline$>60$ & $25,36.76 \%$ & $27,28.42 \%$ & & \\
\hline Education level & & & 3.049 & 0.218 \\
\hline Elementary school or less & $32,47.06 \%$ & $33,34.74 \%$ & & \\
\hline Employment status & & & 3.139 & 0.076 \\
\hline Yes & $32,47.06 \%$ & $58,61.05 \%$ & & \\
\hline No & $36,52.94 \%$ & $37,38.95 \%$ & & \\
\hline Tumor size & & & 6.393 & 0.041 \\
\hline Microadenoma & $9,13.24 \%$ & $22,23.16 \%$ & & \\
\hline Macroadenoma & $54,79.41 \%$ & $72,75.79 \%$ & & \\
\hline Giant adenomas & $5,7.35 \%$ & $1,1.05 \%$ & & \\
\hline Pathological type & & & 2.337 & 0.674 \\
\hline Relapse & & & 1.127 & 0.288 \\
\hline Yes & $11,16.18 \%$ & $10,10.53 \%$ & & \\
\hline No & $57,83.82 \%$ & $85,89.47 \%$ & & \\
\hline
\end{tabular}

PRL, prolactin; PA, pituitary adenoma; $\mathrm{GH}$, growth hormone; ACTH, adrenocorticotropic hormone.

impact on these patients' quality of life.

Measures of quality of life can fully reflect a patient's state of health. In the past, clinicians have focused more on surgical success rate and patient survival rate. Our investigation of quality of life focuses more on patients' wellbeing following surgery and managing long-term complications. The findings of correlational studies investigating postoperative complications and quality of life in patients following $\mathrm{PA}$ resection are currently inconclusive. However, in this study, we demonstrated that the quality of life of these patients with complications is significantly lower than that of patients without complications.

To improve the quality of life of patients that receive neuroendoscopic transnasal PA resections and go on to experience postoperative complications, neurosurgery nurses should constantly update their knowledge and 
Table 3 EQ-5D descriptive system scores for PA patients following surgery (n, \%)

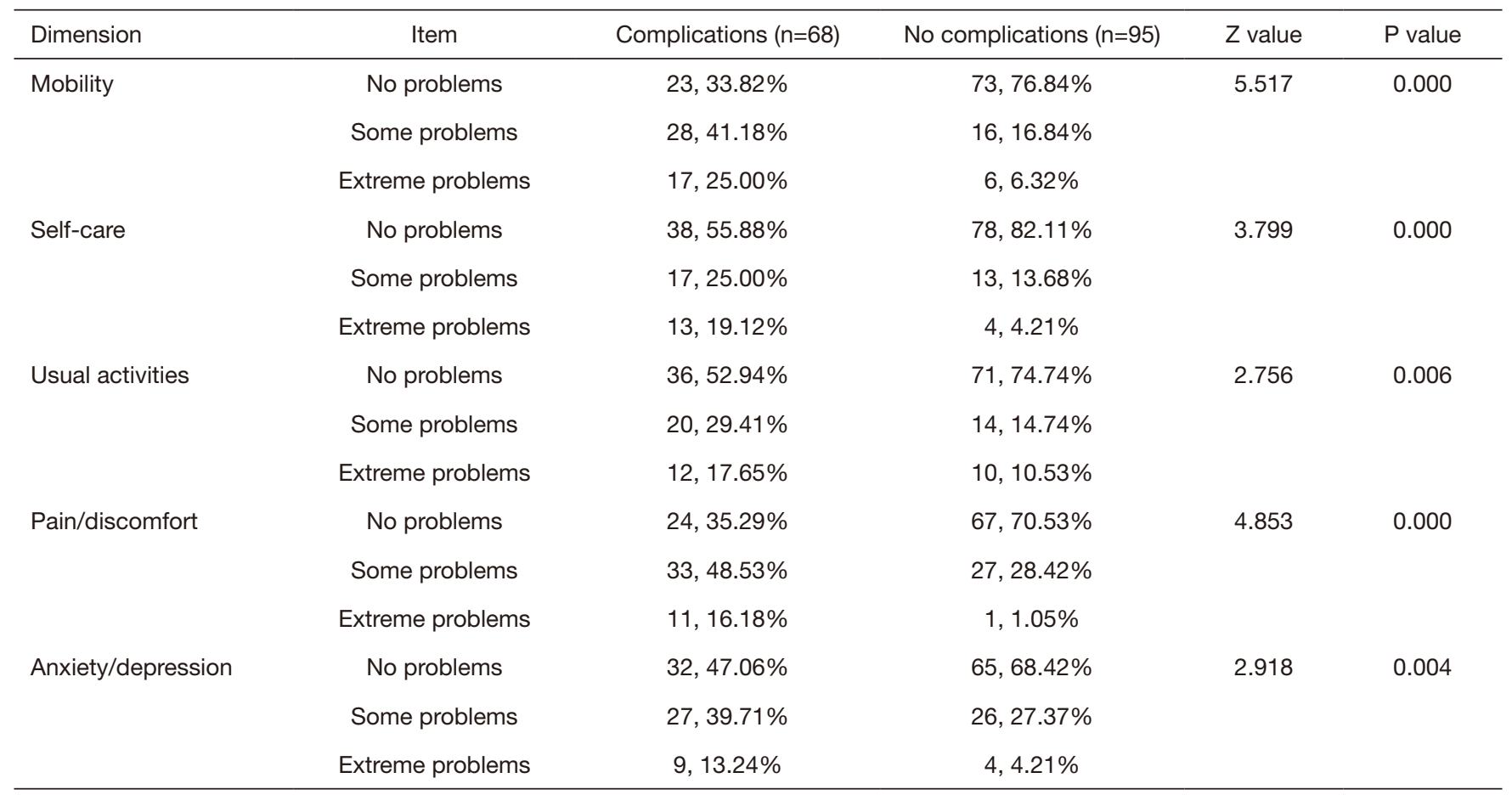

EQ-5D, EuroQol 5 Dimensions; PA, pituitary adenoma.

familiarize themselves with new technologies to strengthen patients' perioperative care. Health education in relation to endoscopy should be provided to patients and their families pre-surgery, and sufficient steps must be taken to prepare patients for surgery, such as nasal cavity preparation. Postsurgery, patients must be closely observed to note potential changes in their condition, and the nasal cavity must be appropriately tended to prevent retrograde infection.

Endoscopy is an important surgical technique that can minimize invasiveness, reduce tumor residue, avoid damage to brain tissue, and more attention should now be paid to the early detection of postoperative complications, such as CSF rhinorrhea and diabetes insipidus. A patient's treatment team must be vigilant for any symptoms of these complications. Elderly patients and patients with larger tumors are high-risk groups of complications, so this group of people should also be paid more attention. All complications have a process of development $(20,21)$ Neurosurgery nurses should constantly update their knowledge, be familiar with the characteristics of new technologies, have disease diagnosis and emergency response capabilities, take preventive nursing measures, prevent complications, and strengthen neuroendoscope transsphenoidal treatment of pituitary glands, including preoperative psychological care, Health education, diet care, nasal cavity care, and observation of the patient's vision and field of vision, etc., can effectively alleviate the psychological burden of patients and improve the effect of surgery. Proper preoperative nasal care is the key to preventing pituitary tumor surgery infection. Nursing staff should carry out strict nasal preparations before surgery for each patient with pituitary tumor and take chloramphenicol eye drops to the patient's abdominal cavity 3-4 days before surgery Instill infusion four times a day. At the same time, the nasal hair inside the patient's nasal cavity should be cleaned and replaced the day before the operation. This process should pay special attention to the protection of the nasal mucosa. Observe the patient's vision and visual field, evaluate the effect of surgical treatment, and observe the changes in the patient's skull. When the patient has sudden visual and visual disturbances, the possibility of intracranial hemorrhage should be considered. Additionally, PA resection patients should be closely examined at followup to appropriately assess and manage any complications. A PA registry should also be established.

This was a single-center retrospective study. The sample 
Table 4 EQ-5D descriptive system scores for PA patients with complications following surgery (n, \%)

\begin{tabular}{|c|c|c|c|c|c|c|}
\hline Dimension & Item & Infection $(n=7)$ & $\begin{array}{l}\text { Hypopituitarism } \\
\qquad(\mathrm{n}=56)\end{array}$ & $\begin{array}{l}\text { CSF rhinorrhea } \\
\qquad(n=18)\end{array}$ & $\begin{array}{l}\text { Diabetes insipidus } \\
\qquad(\mathrm{n}=28)\end{array}$ & $\begin{array}{l}\text { Hyponatremia } \\
\qquad(n=25)\end{array}$ \\
\hline \multirow[t]{3}{*}{ Mobility } & No problems & $1,14.29 \%$ & $16,28.57 \%$ & $5,27.78 \%$ & $10,35.71 \%$ & $11,44.00 \%$ \\
\hline & Extreme problems & $4,57.14 \%$ & $16,28.57 \%$ & $7,38.89 \%$ & $8,28.57 \%$ & $6,24.00 \%$ \\
\hline & $\chi^{2}, \mathrm{P}$ value & $8.846,0.003$ & $33.663,0.000$ & $10.631,0.001$ & $8.714,0.003$ & $3.236,0.072$ \\
\hline \multirow{3}{*}{ Self-care } & Some problems & $1,14.29 \%$ & $15,26.79 \%$ & $2,11.11 \%$ & $4,14.29 \%$ & $3,12.00 \%$ \\
\hline & Extreme problems & $4,57.14 \%$ & $11,19.64 \%$ & $8,44.44 \%$ & $6,21.43 \%$ & $6,24.00 \%$ \\
\hline & $\chi^{2}, P$ value & $9.031,0.003$ & $13.218,0.000$ & $10.640,0.001$ & $1.324,0.250$ & $1.423,0.233$ \\
\hline $\begin{array}{l}\text { Usual } \\
\text { activities }\end{array}$ & $\chi^{2}, P$ value & $8.348,0.004$ & $7.493,0.000$ & $8.153,0.004$ & $0.964,0.326$ & $0.167,0.683$ \\
\hline \multirow{4}{*}{$\begin{array}{l}\text { Pain/ } \\
\text { discomfort }\end{array}$} & No problems & $1,14.29 \%$ & $17,30.36 \%$ & $6,33.33 \%$ & $8,28.57 \%$ & $10,40.00 \%$ \\
\hline & Some problems & $3,42.86 \%$ & $30,53.57 \%$ & $6,33.33 \%$ & $14,50.00 \%$ & $12,48.00 \%$ \\
\hline & Extreme problems & $3,42.86 \%$ & $9,16.07 \%$ & $6,33.33 \%$ & $6,21.43 \%$ & $3,12.00 \%$ \\
\hline & $\chi^{2}, \mathrm{P}$ value & $8.247,0.004$ & $24.694,0.000$ & $8.120,0.004$ & $12.864,0.000$ & $3.170,0.075$ \\
\hline \multirow{2}{*}{$\begin{array}{l}\text { Anxiety/ } \\
\text { depression }\end{array}$} & No problems & $2,28.57 \%$ & $26,46.43 \%$ & $7,38.89 \%$ & $12,42.86 \%$ & $17,68.00 \%$ \\
\hline & Some problems & $4,57.14 \%$ & $22,39.29 \%$ & $7,38.89 \%$ & $10,35.71 \%$ & $5,20.00 \%$ \\
\hline
\end{tabular}

EQ-5D, EuroQol 5 Dimensions; PA, pituitary adenoma; CSF, cerebrospinal fluid.

was somewhat limiting, as cases with missing or inaccurate information were not included in the analyses. This can cause sampling errors. Additionally, the sample size was 163 , which is relatively small. Small sample sizes can impact the results of statistical analyses. It is important, therefore, to conduct future prospective multi-center studies with large sample sizes in this field.

\section{Acknowledgments}

Funding: None.

\section{Footnote}

Reporting Checklist: The authors have completed the STROBE reporting checklist.
Data Sharing Statement: Available at http://dx.doi. org/10.21037/gs-20-690

Conflicts of Interest: All authors have completed the ICMJE uniform disclosure form (available at http://dx.doi. org/10.21037/gs-20-690). The authors have no conflicts of interest to declare.

Ethical Statement: The authors are accountable for all aspects of the work in ensuring that questions related to the accuracy or integrity of any part of the work are appropriately investigated and resolved. The study was conducted in accordance with the Declaration of Helsinki (as revised in 2013). This study was approved by the ethics committee of the Affiliated Hospital of Nantong University ([2017] 02165) and obtained signed informed consent from 
all patients.

Open Access Statement: This is an Open Access article distributed in accordance with the Creative Commons Attribution-NonCommercial-NoDerivs 4.0 International License (CC BY-NC-ND 4.0), which permits the noncommercial replication and distribution of the article with the strict proviso that no changes or edits are made and the original work is properly cited (including links to both the formal publication through the relevant DOI and the license). See: https://creativecommons.org/licenses/by-nc-nd/4.0/.

\section{References}

1. Levy A. Molecular and trophic mechanisms of tumorigenesis. Endocrinol Metab Clin North Am 2008;37:23-50, vii.

2. Theodros D, Patel M, Ruzevick J, et al. Pituitary adenomas: historical perspective, surgical management and future directions. CNS Oncol 2015;4:411-29.

3. Pernicone PJ, Scheithauer BW, Sebo TJ, et al. Pituitary carcinoma: a clinicopathologic study of 15 cases. Cancer 1997;79:804-12.

4. Raappana A, Pirilä T, Ebeling T, et al. Long-term healthrelated quality of life of surgically treated pituitary adenoma patients: a descriptive study. ISRN Endocrinol 2012;2012:675310.

5. The WHOQOL Group. The Development of the World Health Organization Quality of Life Assessment Instrument (the WHOQOL). In: Orley J, Kuyken W. editors. Quality of Life Assessment: International Perspectives. Berlin, Heidelberg: Springer, 1994:41-57.

6. Milian M, Honegger J, Gerlach C, et al. Health-related quality of life and psychiatric symptoms improve effectively within a short time in patients surgically treated for pituitary tumors--a longitudinal study of 106 patients. Acta Neurochir (Wien) 2013;155:1637-45; discussion 1645 .

7. Yang $\mathrm{C}$, Zhang J, Li J, et al. The role of multimodal navigation in endoscopic endonasal surgery for giant pituitary adenomas. Gland Surg 2019;8:663-73.

8. Li J, Shi J, Wang H, et al. Analysis of complications after transnasal resection of pituitary adenoma. Jiangsu Medicine 2008;11:1089-90.

9. Shimon I, Melmed S. Management of pituitary tumors.
Ann Intern Med 1998;129:472-83.

10. Simpson J, Heath J, Wall G. Living with a pituitary tumour: a narrative analysis. Psychol Health 2014;29:162-76.

11. Schipper H, Levitt M. Measuring quality of life: risks and benefits. Cancer Treat Rep 1985;69:1115-25.

12. Levi L. Psychosocial environmental factors and psychosocially mediated effects of physical environmental factors. Scand J Work Environ Health 1997;23 Suppl 3:47-52.

13. Wan C. Discussion on some important issues in the study of quality of life. Chinese Behavioral Medicine 1999;8:66-7.

14. Webb SM. Pituitary tumors: coping with 'cured' pituitary tumors. Nat Rev Endocrinol 2011;7:251-2.

15. Kim SH, Kim HJ, Lee SI, et al. Comparing the psychometric properties of the EQ-5D-3L and EQ-5D-5L in cancer patients in Korea. Qual Life Res 2012;21:1065-73.

16. Kim SH, Hwang JS, Kim TW, et al. Validity and reliability of the EQ-5D for cancer patients in Korea. Support Care Cancer 2012;20:3155-60.

17. Pickard AS, De Leon MC, Kohlmann T, et al. Psychometric comparison of the standard EQ-5D to a 5 level version in cancer patients. Med Care 2007;45:259-63.

18. Chen C, Ding C, Lu G. Study on the correlation between self-perceived aging and quality of life in elderly hypertensive patients in Kaifeng City. Chinese Journal of Practical Nursing 2018;34:2102-5.

19. Day PF, Loto MG, Glerean M, et al. Incidence and prevalence of clinically relevant pituitary adenomas: retrospective cohort study in a Health Management Organization in Buenos Aires, Argentina. Arch Endocrinol Metab 2016;60:554-61.

20. Sun $W$. The application of comfortable nursing during the operation period in the neuroendoscopic single nostril transnasal approach pituitary tumor resection. Journal of Bengbu Medical College 2017;42:1714-15.

21. Yao F, Fan Y. The effect of specialty health education in patients with skull base tumor surgery. Chinese Journal of Practical Nervous Diseases 2016;(1):1-2.

(English Language Editor: B. Madden)

Cite this article as: Zhang J, Wang $\mathrm{Y}, \mathrm{Xu} \mathrm{X}$, Gu Y, Huang F, Zhang M, Xu X, Gu Y. Postoperative complications and quality of life in patients with pituitary adenoma. Gland Surg 2020;9(5):1521-1529. doi: 10.21037/gs-20-690 